\title{
Hormones and Dry Eye Disease
}

Juan Ding*

Department of Ophthalmology, Harvard Medical School, USA

*Corresponding author: Juan Ding, Schepens Eye Research Institute, Massachusetts Eye and Ear, Department of Ophthalmology, Harvard Medical School, 20 Stanford St., Boston, MA 02114, USA, Tel: 617-912-0288; E-mail: Juan_ding@meei.harvard.edu

Received date: August 06, 2014, Accepted date: August 08, 2014, Published date: August 15, 2014

Copyright: (c) 2014 Ding J. This is an open-access article distributed under the terms of the Creative Commons Attribution License, which permits unrestricted use, distribution, and reproduction in any medium, provided the original author and source are credited.

\section{Introduction}

Dry eye disease (DED) is a very prevalent eye disorder, affecting 40 million people in the United States alone. The primary cause of DED is meibomian gland dysfunction (MGD) [1], which by itself is a disease entity. The meibomian gland is a large sebaceous gland located in the tarsal plate of the eye lids. It functions to synthesize and secrete lipids onto the ocular surface. These lipids make up the outer-most layer in the tear film and reduce tear evaporation. When the quantity or quality of the lipids is compromised such as occurs in MGD, tears evaporate more quickly, leading to increased DED, ocular surface discomfort, impaired vision and decreased quality of life.

Several risk factors for MGD have been identified, including aging, the female sex, androgen deficiency, rosacea, and medications such as isotretinoin (13-cis retinoic acid), antiandrogens and postmenapausal hormone replacement therapy [2]. In understanding why these factors are risk factors for MGD, one particularly interesting aspect is the hormonal regulation, as skin sebaceous glands are known to be heavily affected by hormones such as androgens, growth hormone (GH), insulin, insulin-like growth factor-1 (IGF-1), etc. [1]. These studies are made possible by a human sebaceous gland cell line. Recently, Dr. David A Sullivan's laboratory from Boston, Massachusetts has developed an immortalized human meibomian gland epithelial cell line using human telomerase reverse transcriptase. This cell line shows a normal karyotype, similar gene expression profiles to primary human meibomian gland epithelial cells, and can be induced to proliferate or differentiate under specific culture conditions [3-5]. This cell line has been sent to research laboratories all over the world for meibomian gland studies. In addition, we have created an in vitro MGD model using this cell line exposed to 13-cis retinoic acid, which induces cellular phenotypes and gene expression profiles characteristics of MGD. With the establishment of this cell line and the MGD model, we have started revealing the nature of the endocrine regulation of human meibomian gland and have identified possible interventions to counteract MGD. Here we will briefly summarize our recent findings related to hormones.

\section{Isotretinoin}

This is not a hormone per se, but it is often used systemically and is a risk factor for MGD. Isotretinoin, a vitamin A derivative, is used to treat severe acne; it is also a chemotherapeutic drug to treat cancer, and frequently found in anti-aging cosmetics due to its wrinklereducing effect. While quite effective in suppressing skin sebaceous gland growth, differentiation and lipid secretion, it also exerts a similar action on the meibomian gland, leading to dry eye. To understand the cellular and molecular mechanism of this action, we applied isotretinoin to immortalized human meibomian gland epithelial cells and analyzed the cellular phenotypes [6]. We discovered that isotretinoin inhibits cell proliferation, induces cell death, and significantly alters the expression of more than six thousand genes, including those involved in cell proliferation, cell death, differentiation, keratinisation and inflammation; further, isotretinoin also inhibits Akt signalling and increases the generation of interleukin-1 $\beta$ and matrix metallopeptidase 9 , both known to be associated with dry eye disease [7]. This demonstrates that vitamin A, even though an essential and beneficial factor for the health of the ocular surface, is toxic to the meibomian gland and induces dry eye at high doses. Therefore clinicians should monitor the dry eye side effect when treating acne or cancer using isotretinoin. Isotretinoin-exposed meibomian gland cells show characteristics of MGD, including cell atrophy and keratinisation, therefore we propose that this can be used as a model to study MGD pathophysiology and test treatments that may alleviate MGD in vitro.

\section{Growth Hormone and IGF-1}

The GH/IGF-1 axis promotes sebaceous gland proliferation and lipid secretion [8]. This leads to our hypothesis that GH and IGF-1 will similarly act on the meibomian gland. In support of this hypothesis, we have found that IGF-1 increases meibomian gland cell proliferation and lipid accumulation, and blocking the IGF-1 receptor diminishes these effects of IGF-1; however, GH does not produce an obvious phenotype in these cells [9]. We are actively seeking to identify the effect of GH on the meibomian gland in vivo and in co-culture models where the meibomian gland epithelial cells are cultured together with other supporting cell types that are present in vivo. The finding of IGF-1 receptor on the meibomian gland is clinically significant, as a cancer drug targeting IGF-1 receptor has already been found to lead to dry eye side effect in healthy human subjects [10]. Further, IGF-1 receptor is also considered a target for thyroid eye disease $[11,12]$. Therefore ophthalmologists should monitor meibomian gland health and dry eye disease when treating patients with IGF-1 receptor blockers.

\section{And More}

We are currently also working to elucidate the actions of steroid hormones such as androgens and estrogens, as well as insulin, a hormone that is dysregulated in diabetes. There are more than 50 hormones in the human body, and we are only begining to understand the endocrine regulation of the human meibomian gland. We welcome scientists all over the world to study the biology of this gland that plays such an essential role for ocular surface health.

\section{References}

1. Knop E, Knop N, Millar T, Obata H, Sullivan DA (2011) The international workshop on meibomian gland dysfunction: report of the 
subcommittee on anatomy, physiology, and pathophysiology of the meibomian gland. Invest Ophthalmol Vis Sci 52: 1938-1978.

2. Schaumberg DA, Nichols JJ, Papas EB, Tong L, Uchino M, et al. (2011) The international workshop on meibomian gland dysfunction: report of the subcommittee on the epidemiology of, and associated risk factors for, MGD. Invest Ophthalmol Vis Sci 52: 1994-2005.

3. Liu S, Hatton MP, Khandelwal P, Sullivan DA (2010) Culture, immortalization, and characterization of human meibomian gland epithelial cells. Invest Ophthalmol Vis Sci 51: 3993-4005.

4. Liu S, Richards SM, Lo K, Hatton M, Fay A, et al. (2011) Changes in gene expression in human meibomian gland dysfunction. Invest Ophthalmol Vis Sci 52: 2727-2740.

5. Liu S, Kam WR, Ding J, Hatton MP, Sullivan DA (2013) Effect of growth factors on the proliferation and gene expression of human meibomian gland epithelial cells. Invest Ophthalmol Vis Sci 54: 2541-2550.

6. Ding J, Kam WR, Dieckow J, Sullivan DA (2013) The influence of 13-cis retinoic acid on human meibomian gland epithelial cells. Invest Ophthalmol Vis Sci 54: 4341-4350.

7. [No authors listed] (2007) The epidemiology of dry eye disease: report of the Epidemiology Subcommittee of the International Dry Eye WorkShop (2007). Ocul Surf 5: 93-107.
8. Deplewski D, Rosenfield RL (1999) Growth hormone and insulin-like growth factors have different effects on sebaceous cell growth and differentiation. Endocrinology 140: 4089-4094.

9. Ding J, Sullivan DA (2014) The effects of insulin-like growth factor 1 and growth hormone on human meibomian gland epithelial cells. JAMA Ophthalmol 132: 593-599.

10. Yin D, Sleight B, Alvey C, Hansson AG, Bello A (2013) Pharmacokinetics and pharmacodynamics of figitumumab, a monoclonal antibody targeting the insulin-like growth factor 1 receptor, in healthy participants. J Clin Pharmacol 53: 21-28.

11. Smith TJ, Hegedüs L, Douglas RS (2012) Role of insulin-like growth factor-1 (IGF-1) pathway in the pathogenesis of Graves' orbitopathy. Best Pract Res Clin Endocrinol Metab 26: 291-302.

12. Wiersinga WM (2011) Autoimmunity in Graves' ophthalmopathy: the result of an unfortunate marriage between TSH receptors and IGF-1 receptors? J Clin Endocrinol Metab 96: 2386-2394. 